\title{
Confinement From The Gauge Invariant Abelian Decomposition
}

\author{
Nigel Cundy* \\ Lattice Gauge Theory Research Center, FPRD, and CTP, Department of Physics \& Astronomy, \\ Seoul National University, Seoul, 151-747, South Korea \\ E-mail: ndcundy@phya.snu.ac.kr
}

\section{Yongmin Cho}

Administration Building 310-4, Konkuk University, Seoul 143-701, Korea

\section{Weonjong Lee}

Lattice Gauge Theory Research Center, FPRD, and CTP, Department of Physics \& Astronomy, Seoul National University, Seoul, 151-747, South Korea

\begin{abstract}
A common approach while considering confinement is to study the dominance of an Abelian subgroup of the SU(3) gauge Links. A good way to find the Abelian component of the field is through the Cho-Guan-De gauge invariant Abelian Decomposition, which uses a carefully chosen direction vector $n$ to split the gauge field into an Abelian restricted field and a remnant coloured field. The restricted field can be further subdivided into topological and non-topological terms. We show that there is a choice of $n$ which allows us to exactly represent the Wilson Loop of full QCD as a function of only the restricted Abelian field without requiring any path ordering or additional path integrals. We present numerical evidence showing that the topological part of the restricted field dominates the string tension. We also show that $n$ contains certain topological objects, which, if they exist, will be at least partially responsible for confinement. These leave distinctive patterns in the restricted field strength, and we search for these structures in quenched lattice QCD.
\end{abstract}

31st International Symposium on Lattice Field Theory - LATTICE 2013

July 29 - August 3, 2013

Mainz, Germany

*Speaker. 


\section{Introduction}

We [1] seek to explain the emergence of the linear string tension in QCD by studying the Wilson Loop. A common approach, often achieved by fixing to a particular gauge [2], is to extract the Abelian part of the gauge link (Abelian decomposition), projecting out the coloured, off-diagonal, elements of the gauge link leaving just an Abelian, colour neutral field which is expected to dominate confinement. It is best to respect gauge invariance by using the Cho-Duan-Ge (CDG) Abelian decomposition [3]. The CDG decomposition extracts the components of the gauge field aligned with $N_{C}-1$ commuting traceless colour vectors $n_{j}$; our choice of $n_{j}$ is the main novelty of this study. Other recent studies [4] select $n$ (the Abelian theory must be U(1) in that work) through additional dynamical fields which allow the authors to relate the string tensions of the Abelian theory and full QCD. Instead, we maximise the Abelian symmetry so that all the possible degrees of freedom are included. It is possible to choose a specific $n_{j}$ which diagonalises the Wilson Loop and leaves the maximal $U(1)^{N_{C}-1}$ Abelian symmetry for an original $\mathrm{SU}\left(N_{C}\right)$ gauge theory. This $U(1)^{N_{C}-1}$ theory can be studied numerically and modelled theoretically. We believe that certain topological objects contained within the colour fields can provide an explanation of confinement.

In section 2, we use a particular choice of the CDG decomposition to diagonalise the Wilson Loop, and outline how this might be used to demonstrate a linear static potential. In section 3, we provide numerical results supporting our analysis, and we conclude in section 4 .

\section{Diagonalisation of Wilson Loops}

A linear static potential, $V(R)$, is a signal for confinement. For a gauge field $A_{\mu}, V(R)$ may be constructed using $V(R)=-\lim _{T \rightarrow \infty} \frac{1}{T} \log \left\langle\operatorname{tr} W[\{R, T\}, U] / N_{C}\right\rangle$, where $W$ is the $R \times T$ Wilson Loop [5]. Consider a Wilson loop of length $L=N \delta \sigma$ parametrised by $\sigma$ around a curve $C_{s}$, a $R \times T$ rectangle in the $x$-t plane, with $x_{\mu}(\sigma=0)=x_{\mu}(\sigma=L)=s_{\mu}$, where $P$ represents path ordering,

$$
W\left[C_{s}, U\right]=\lim _{\delta \sigma \rightarrow 0} \prod_{\sigma=0, \delta \sigma, 2 \delta \sigma, \ldots}^{(N-1) \delta \sigma} U_{\mu(\sigma)}(x(\sigma)) \quad U_{\mu}(x)=P\left[e^{-i g \int_{x}^{x+\delta \sigma \hat{\mu}} d x_{\mu}^{\prime} A_{\mu}}\right] .
$$

We now insert an identity operator $I_{\sigma}^{r}=\theta_{\sigma}^{r}\left(\theta_{\sigma}^{r}\right)^{-1}$ between each pair of gauge links, with $\theta \in$ $U\left(N_{C}\right)$ and $r$ an index identifying the Wilson Loop. We choose $\theta$ so that it diagonalises the gauge links along the Wilson Loop. The index $j$ runs over only the diagonal Gell-Mann matrices.

$$
\begin{aligned}
\theta_{s}^{\dagger} W\left[C_{s}\right] \theta_{s} & =\lim _{\delta \sigma \rightarrow 0} \prod_{\sigma=0, \delta \sigma, 2 \delta \sigma, \ldots}^{(N-1) \delta \sigma} \theta_{\sigma}^{\dagger} U_{\mu(\sigma)}(x(\sigma)) \theta_{\sigma+\delta \sigma} \\
{\left[\theta_{\sigma}^{\dagger} U_{\mu(\sigma)}(x(\sigma)) \theta_{\sigma+\delta \sigma}, \lambda^{j}\right] } & =0, \quad \lambda^{j} \text { diagonal Gell-Mann matrix, } \forall j, U_{\mu} \in C_{s} .
\end{aligned}
$$

$\theta$ is uniquely defined up to a $U(1)^{N_{C}}$ transformation $\chi$ (i.e. $\theta \rightarrow \theta \chi$ ) and the ordering of the eigenvectors. By diagonalising the gauge links on all nested Wilson Loops, we can extend this definition of $\theta$ across the entire lattice. We now introduce new $S U\left(N_{C}\right)$ fields, $\hat{U}$ and $\hat{X}$, so that

$$
\left[\theta_{\sigma}^{\dagger} \hat{U}_{\mu(\sigma)}(x(\sigma)) \theta_{\sigma+\delta \sigma}, \lambda^{j}\right]=0 \quad \forall x, \mu, j ; \quad U_{\mu}(x)=\hat{X}_{\mu} \hat{U}_{\mu} \quad \forall x, \mu ; \quad \hat{U}_{\mu}(x)=U_{\mu}(x) \forall x, \mu \in C_{s} .
$$


This allows us to express the Wilson Loop without any path ordering,

$$
\hat{U}_{\mu}(x)=\theta_{x} e^{-i \int_{x}^{x+\delta \sigma \hat{\mu}} d x_{\mu}^{\prime} \hat{u}_{\mu}^{j}\left(x^{\prime}\right) \lambda^{j}} \theta_{x+\delta \sigma \hat{\mu}}^{\dagger} ; \quad \operatorname{tr} W\left[C_{s}, U\right]=\operatorname{tr} W\left[C_{S}, \hat{U}\right]=\operatorname{tr} e^{-i g \oint_{C_{S}} d x^{\mu} \lambda^{j} \hat{u}_{\mu}^{j}(x)} .
$$

We can extract the string tension from the Abelian field $\hat{u}$ (a function of $\theta$ and $A_{\mu}$ ). We use Stoke's theorem to express the line integral over the Abelian field as a surface integral.

$$
\oint_{C_{s}} d x^{\mu} \hat{u}_{\mu, x}^{j}=\int_{x \in \Sigma, x \notin \tilde{\Sigma}} d \Sigma^{\mu v} \hat{F}_{\mu \nu}^{j}+\sum_{n=1}^{\tilde{N}} \oint_{\tilde{C}_{n}} d x^{\mu} \hat{u}_{\mu, x}^{j} ; \quad \hat{F}_{\mu \nu}^{j}=\partial_{\mu} \hat{u}_{v}^{j}-\partial_{v} \hat{u}_{\mu}^{j} .
$$

$\Sigma$ represents the planar surface bound by $C_{s} . \tilde{\Sigma}_{n}$ represents the $\tilde{N}$ regions within $\Sigma$ (bound by the curves $\tilde{C}_{n} \in \Sigma$ ) where $\hat{u}$ is not analytic. $\hat{F}$ and $\hat{u}$ are gauge invariant. Defining $X_{0}=\frac{1}{2}\left(\hat{X}+\hat{X}^{\dagger}\right)$,

$$
\begin{gathered}
i \delta \tilde{\sigma} \hat{u}_{\mu, x}^{j}=\frac{1}{\operatorname{tr}\left(\lambda^{j}\right)^{2}} \operatorname{Im}\left(\operatorname{tr}\left[\lambda^{j} \theta_{x}^{\dagger} \hat{X}_{\mu, x}^{\dagger} \theta_{x} \theta_{x}^{\dagger} U_{\mu, x} \theta_{x+\delta \tilde{\sigma} \hat{\mu}]}\right)\right. \\
=\frac{1}{2 \operatorname{tr}\left(\lambda^{j}\right)^{2}} \operatorname{tr}\left[\lambda^{j} \theta_{x}^{\dagger}\left(\hat{X}_{\mu, x}^{\dagger}-\hat{X}_{\mu, x}\right) \theta_{x}-2 i \lambda^{j} \delta \tilde{\sigma} \theta_{x}^{\dagger}\left[X_{0}\right]_{\mu, x} g A_{\mu, x}^{a} \lambda^{a} \theta_{x}+\right. \\
\left.\quad 2 \lambda^{j} \theta^{\dagger}\left[X_{0}\right]_{\mu, x} \theta_{x} \delta \tilde{\sigma} \theta_{x}^{\dagger} \partial_{\tilde{\sigma}} \theta\right]+O\left(\delta \sigma^{2}\right) .
\end{gathered}
$$

We choose $\hat{X}$ so that $\operatorname{tr}\left(\lambda^{j} \theta_{x}^{\dagger}\left(\hat{X}_{\mu}(x)-\hat{X}_{\mu}(x)^{\dagger}\right) \theta_{x}\right)=0$ and $\operatorname{tr} \hat{X}_{\mu}(x)$ is maximised. If the singularity in $\hat{u}$ occurs over a small region where $A_{\mu}$ and $X_{0}$ are smooth, the $\theta^{\dagger} \partial_{\mu} \theta$ term will dominate, and

$$
\oint_{C_{s}} d x^{\mu} \hat{u}_{\mu}(x)^{j}=\sum_{n} \oint_{\tilde{C}_{n}} d \tilde{\sigma} \operatorname{tr}\left[\lambda^{j} \theta^{\dagger}\left[X_{0}\right]_{\mu, x} \theta_{x} \delta \tilde{\sigma} \theta_{x}^{\dagger} \partial_{\tilde{\sigma}} \theta\right]+\ldots
$$

From the above analysis, we see that $\hat{U}$ and $\hat{X}$ are uniquely defined by the equations

$$
\hat{U}_{\mu, x} n_{j, x+\delta \sigma \hat{\mu}} \hat{U}_{\mu, x}^{\dagger}-n_{j, x}=0 \quad \operatorname{tr} n^{j}\left(\hat{X}-\hat{X}^{\dagger}\right)=0 \quad n_{j, x} \equiv \theta_{x} \lambda^{j} \theta_{x}^{\dagger} .
$$

This is a lattice representation of the continuum Cho-Duan-Ge gauge-invariant Abelian decomposition [3], which is known to contain topological singularities within the colour field $n$.

Non-analyticities in the $\theta$ field occur when (a) the Wilson Loop has degenerate eigenvalues; (b) $A_{\mu}$ is discontinuous (in the chosen gauge); or (c) the situation described below. After gauge fixing, for a $\mathrm{SU}(2)$ theory, we parametrise $\theta$ using a complex Givens rotation and a $\mathrm{U}(1)$ term,

$$
\theta=e^{i a \phi} e^{i d_{3} \lambda^{3}} ; \quad \phi=\left(\begin{array}{cc}
0 & e^{i c} \\
e^{-i c} & 0
\end{array}\right) ; \quad \bar{\phi}=\left(\begin{array}{cc}
0 & i e^{i c} \\
-i e^{-i c} & 0
\end{array}\right) \text {. }
$$

$a, c$ and $d_{3}$ are not gauge invariant; $0 \leq a \leq \pi / 2, c, d_{3} \in \mathbb{R}$. In SU(3), we construct $\theta$ from three Givens terms and a $U(1) \times U(1)$ matrix parametrised by $d_{3}$ and $d_{8}$. The arbitrary parameters $d_{3}$ and $d_{8}$ do not affect the field $n$ (they can be chosen to be zero). $c$ is ill defined at $a=0$ or $a=\pi / 2$. We parametrise space-time around one of these points as

$$
(t, x, y, z) \equiv r\left(\cos \psi_{3}, \sin \psi_{3} \cos \psi_{2}, \sin \psi_{3} \sin \psi_{2} \cos \psi_{1}, \sin \psi_{3} \sin \psi_{2} \sin \psi_{1}\right),
$$

with $r=0$ at $a=\pi / 2$. In SU(2), by writing $c=v_{n} \psi_{3}$ for an integer gauge-invariant winding number $v_{n} \neq 0$, and using $\theta^{\dagger} \partial_{\sigma} \theta=e^{-i d_{3} \lambda^{3}}\left[i \partial_{\sigma} a \phi+i \lambda^{3} \partial_{\sigma} d+i \sin a \cos a \bar{\phi} \partial_{\sigma} c-i \sin ^{2} a \partial_{\sigma} c \lambda^{3}\right] e^{i d_{3} \lambda^{3}}$, we may integrate around a curve at fixed $a=a_{0 n}$ surrounding the structure in $\hat{F}$ to obtain

$$
\oint_{C_{s}} d x^{\mu} \hat{u}_{\mu}(x)^{j}=\sum_{n=1}^{\tilde{N}} 2 \pi v_{n} \sin ^{2} a_{0 n} \operatorname{tr}\left[\left[X_{0}\right]_{\mu, x}\right]+\ldots
$$


If the number of structures, $\tilde{N}$, within the Wilson loop is proportional to the area enclosed by the loop, as might be expected, then this leads to an area law string tension and confinement.

We parametrise $a=\frac{\pi}{2}-G\left(r, \psi_{1}, \psi_{2}, \psi_{3}\right)$ and $c=J\left(\psi_{3}\right)$ for unknown gauge-dependent functions $G$ and $J$. Then, we can calculate the topological $(\theta)$ contribution $H_{\mu \nu}^{3}$ to the field strength $\hat{F}_{\mu \nu}^{3} . H_{\mu \nu}^{j}=\frac{1}{8 g} \operatorname{tr} n_{j}\left[\partial_{\mu} n_{k}, \partial_{v} n_{k}\right]$. In SU(2), with $G \sim \partial_{i} G \equiv \frac{\partial G}{\partial \psi_{i}} \sim r^{\xi} ; \partial_{r} G \sim r^{\xi-1}$ and $\xi>0$

$$
\begin{aligned}
& B_{y}=\frac{1}{g} \sin 2 G(\underbrace{\partial_{1} G \partial_{3} J \frac{y x t}{r^{2} r_{x y z} r_{y z}^{2}}}_{\text {t-string }}+\underbrace{\partial_{2} G \partial_{3} J \frac{z t}{r^{2} r_{x y z} r_{y z}}}_{\text {t-string }}) \quad B_{x}=\frac{1}{g} \sin 2 G(\underbrace{\partial_{1} G \partial_{3} J \frac{t}{r_{x y z} r^{2}}}_{\text {t-string }}) \\
& E_{x}=-\frac{1}{g} \sin 2 G(\underbrace{\partial_{r} G \partial_{3} J \frac{x}{r r_{x y z}}}_{\text {point }}-\underbrace{\partial_{2} G \partial_{3} J \frac{r_{y z}}{r^{2} r_{x y z}}}_{\text {point }}) \quad B_{z}=-\frac{1}{g} \sin 2 G(\underbrace{\partial_{1} G \partial_{3} J \frac{z x t}{r^{2} r_{y z}^{2} r_{x y z}}}_{\mathrm{t} \text {-string }}) \\
& E_{y}=-\frac{1}{g} \sin 2 G(\underbrace{\partial_{r} G \partial_{3} J \frac{y}{r_{x y z} r}}_{\text {point }}-\underbrace{\partial_{1} G \partial_{3} J \frac{z r_{x y z}}{r^{2} r_{y z}^{2}}}_{\text {x-string }}+\underbrace{\partial_{2} G \partial_{3} J \frac{x y}{r^{2} r_{x y z} r_{y z}}}_{\text {point }}) \\
& E_{z}=-\frac{1}{g} \sin 2 G(\underbrace{\partial_{r} G \partial_{3} J \frac{z}{r r_{x y z}}}_{\text {point }}-\underbrace{\partial_{1} G \partial_{3} J \frac{y r_{x y z}}{r^{2} r_{y z}^{2}}}_{\text {x-string }}-\underbrace{\partial_{2} G \partial_{3} J \frac{z x}{r_{y z} r_{x y z} r^{2}}}_{\text {point }}) .
\end{aligned}
$$

a $\mu$-string is a 1-Dimensional object parallel to the $\mu$-axis; a point is a structure where the maximum falls at least as $1 / r$ in all directions (for $\xi=1$ ). After rotating the coordinate system consistent with the overall symmetry, we find the following structures in the electromagnetic field strength:

\begin{tabular}{lllllll}
\hline Parametrisation & $E_{x}$ & $E_{y}$ & $E_{z}$ & $B_{x}$ & $B_{y}$ & $B_{z}$ \\
\hline Equation (2.8) & point & $\mathrm{x}$-string & $\mathrm{x}$-string & $\mathrm{t}$-string & $\mathrm{t}$-string & $\mathrm{t}$-string \\
$t \leftrightarrow x$ & point & $\mathrm{x}$-string & $\mathrm{x}$-string & $\mathrm{x}$-string & $\mathrm{t}$-string & $\mathrm{t}$-string \\
$y \leftrightarrow z$ & point & $\mathrm{x}$-string & $\mathrm{x}$-string & $\mathrm{t}$-string & $\mathrm{t}$-string & $\mathrm{t}$-string \\
$t \leftrightarrow x, y \leftrightarrow z$ & point & $\mathrm{x}$-string & $\mathrm{x}$-string & $\mathrm{x}$-string & $\mathrm{t}$-string & $\mathrm{t}$-string \\
\hline \hline
\end{tabular}

If these topological structures exist, they will reveal themselves as points in the $x t$ component of the field strength, and either points, $x$-strings or $t$-strings in the other components of the field strength.

\section{Numerical Results}

We used quenched Luscher-Weisz [6] lattice QCD configurations. Our Lattice spacings and lattice volumes are shown in table 1. To preserve gauge invariance, we use a stout smeared gauge field $\tilde{U}_{p}$ (after a large number, $p$, of smearing steps) during our construction of the topological field, $M$, taken from the Abelian decomposition of $\tilde{M}_{p}=\theta^{\dagger} \tilde{U}_{p} \theta ; \tilde{U}$ will not contribute to the string tension. In figure 1 and table 2 we show results for the string tension for the gauge fields $U, \hat{U}$, and $M$, the topological $(\theta)$ part of $\hat{U}$. To save computer time, initial results used a single $\theta$ for each Wilson Loop on a configuration, breaking the identity between $\operatorname{tr} W\left[C_{s}, U\right]$ and $\operatorname{tr} W\left[C_{s}, \hat{U}\right]$. The $\hat{U}$ and $M$ string tensions (seen in the slope of the curves) are in good agreement.

Figure 2 displays contour plots showing the distribution of the various components of the field strength. The dominant structures appear to be points or lines in the expected directions. This 


\begin{tabular}{l|lllll}
\hline Name & Lattice size & $\mathrm{L}(\mathrm{fm})$ & $\beta$ & $a(\mathrm{fm})$ & $\#$ \\
\hline 8.0 & $16^{3} \times 32$ & 2.30 & 8.0 & $0.144(2)$ & 91 \\
8.3 & $16^{3} \times 32$ & 1.84 & 8.3 & $0.114(1)$ & 91 \\
8.52 & $16^{3} \times 32$ & 1.58 & 8.52 & $0.099(1)$ & 82 \\
$8.3 \mathrm{~L}$ & $20^{3} \times 40$ & 2.30 & 8.3 & $0.112(5)$ & 54 \\
\hline \hline
\end{tabular}

Table 1: Parameters for the ensembles. \# is the number of configurations. $L$ the physical spatial extent.

\begin{tabular}{l|llllllll}
\hline & $U$ & $\hat{U}$ & $\tilde{U}_{600}$ & $M_{600}$ & $\tilde{U}_{800}$ & $M_{800}$ & $\tilde{U}_{1000}$ & $M_{1000}$ \\
\hline 8.0 & $0.094(2)$ & $0.116(4)$ & $0.0273(2)$ & $0.103(10)$ & $0.0213(1)$ & $0.104(8)$ & $0.0174(1)$ & $0.105(9)$ \\
8.3 & $0.0590(8)$ & $0.095(2)$ & $0.0185(1)$ & $0.087(5)$ & $0.0147(1)$ & $0.087(5)$ & $0.0122(1)$ & $0.087(5)$ \\
8.52 & $0.0442(6)$ & $0.077(1)$ & $0.0149(2)$ & $0.076(3)$ & $0.0124(2)$ & $0.076(3)$ & $0.0106(2)$ & $0.077(3)$ \\
$8.3 \mathrm{~L}$ & $0.057(5)$ & $0.099(1)$ & $0.0179(1)$ & $0.099(2)$ & $0.0144(1)$ & $0.099(2)$ & $0.0121(1)$ & $0.098(2)$ \\
\hline \hline
\end{tabular}

Table 2: The string tension for the ensembles ( $\theta$ fixed/configuration)
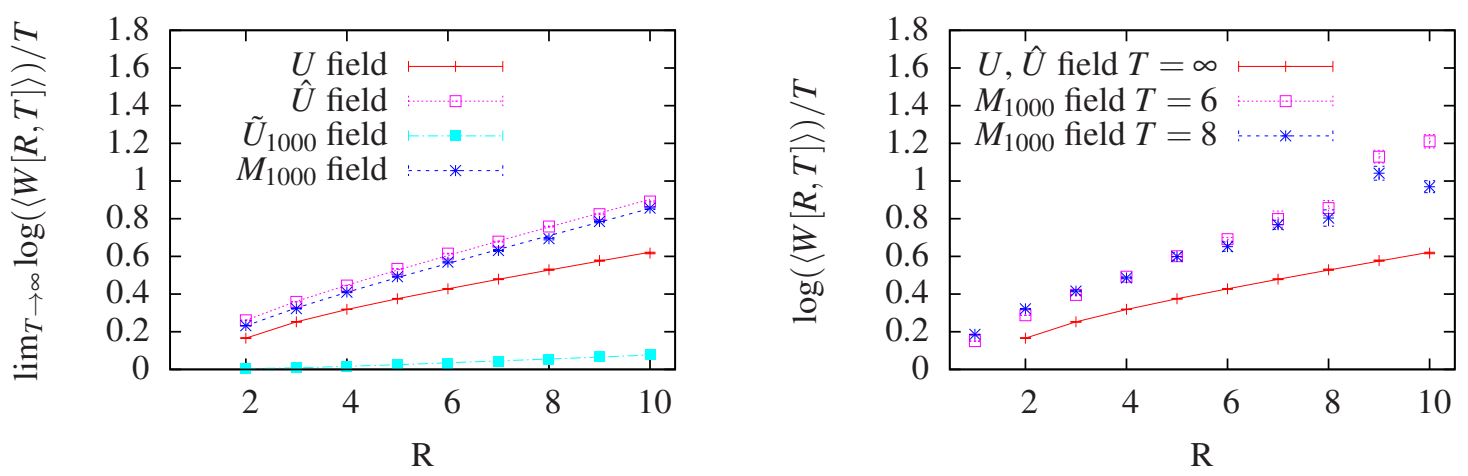

Figure 1: The string tension calculated from (R,T) Wilson Loops on the $\beta=8.52$ ensemble, for $\theta$ is fixed for each configuration (left) and early results with $\theta$ recalculated for each Wilson Loop (right). Our data where $\theta$ is recalculated is not yet good enough for a reliable extrapolation to $T=\infty$.
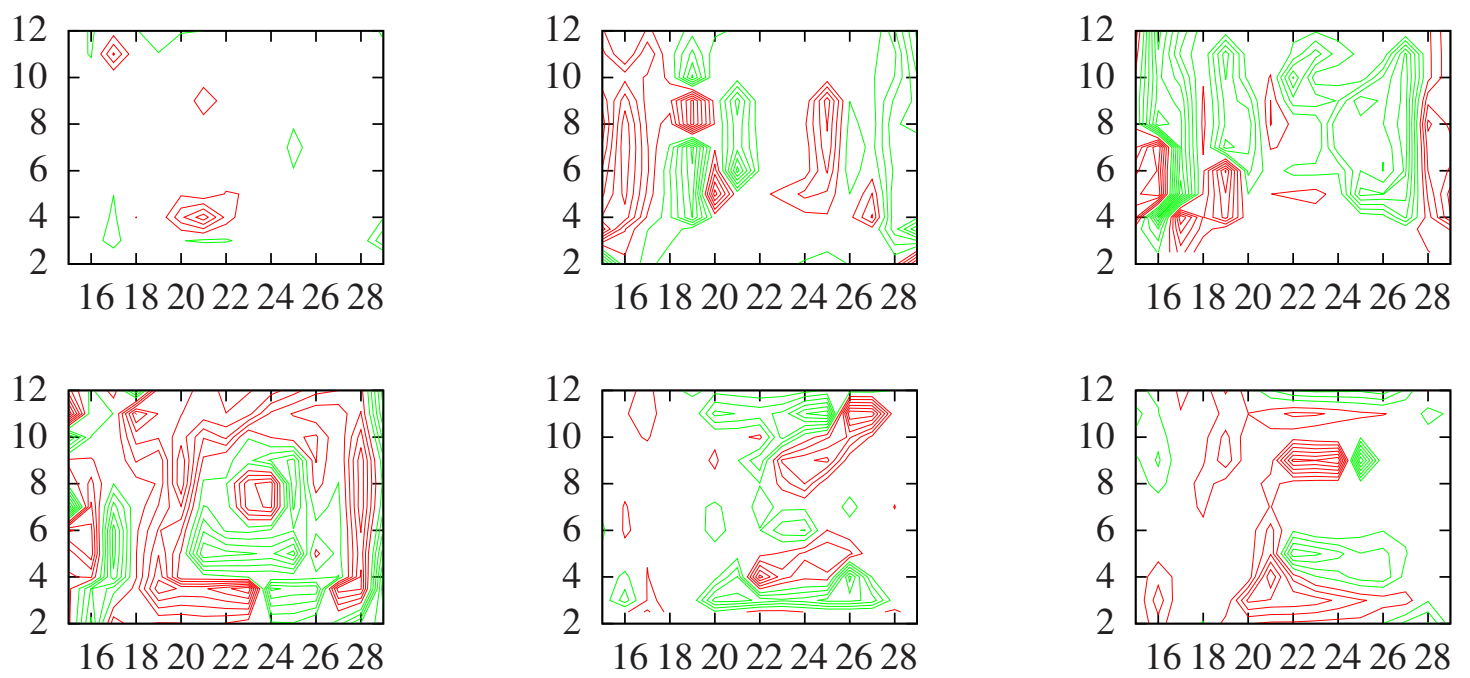

Figure 2: Contour plots for the field strength for the $x$ (left), $y$ (middle) and $z$ (right) components of the restricted electric (top) and magnetic (bottom) fields on an X (y-axis)-T (x-axis) planar slice of the lattice. Red indicates positive field strength, green negative field strength. 

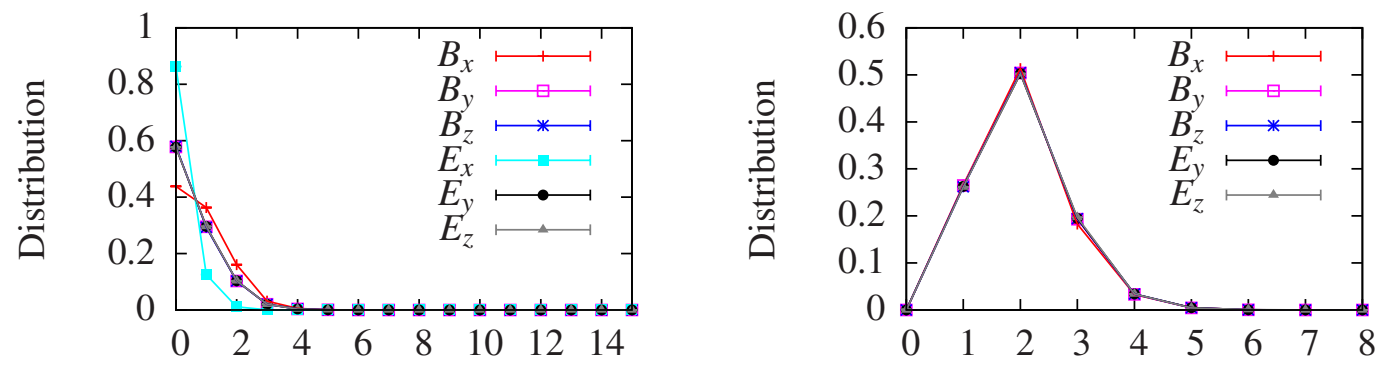

Figure 3: The average number of nearest neighbours within a cluster for each lattice site in the cluster (left) and the same analysis only including points within clusters extended over at least four lattice sites (right).
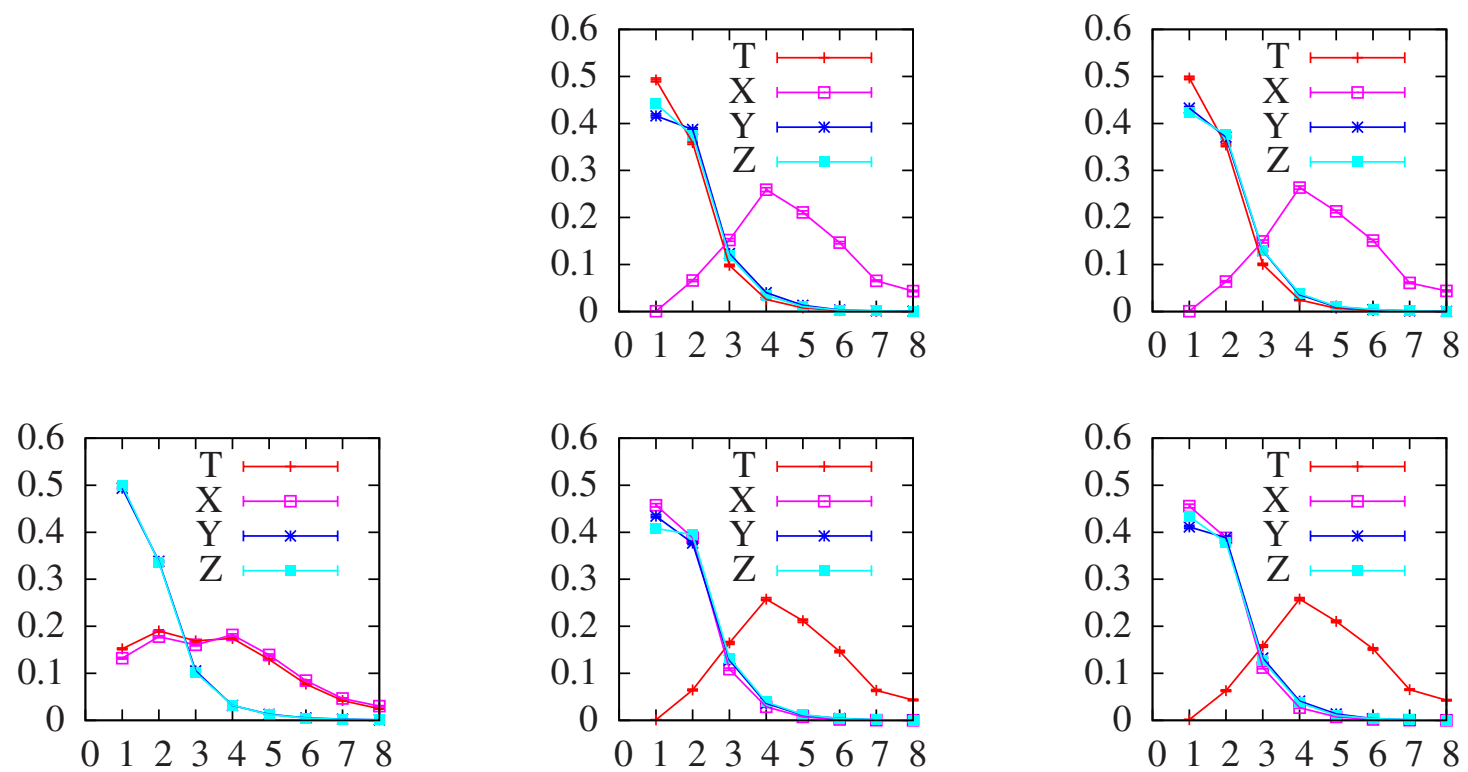

Figure 4: The spatial extent of the clusters containing more than four lattice sites along the four spatial directions for the $x$ (left) $y$ (middle) and $z$ (right) components of the electric (top) and magnetic (bottom) fields. The $X$ axis gives the length of the cluster in a given direction; the $Y$ axis the proportion of clusters with that length.

is confirmed by a cluster analysis. We identify clusters as sign-coherent regions of field strength with $\left|\hat{F}_{\mu \nu}\right|>1$ for each $\mu, v$. We then compare the size, shape and orientation of the clusters with the model expectations of the topological objects in the field strength. In figures 3 and 4 , we investigate whether the objects of within the Abelian Field strength have the shapes expected from the theory. Figure 3 investigates the dimensionality of the clusters by investigating the number of nearest neighbours of each site in the cluster. Excluding the smallest clusters, the majority of lattice sites have two neighbouring sites within the same cluster, suggesting that these objects are one-dimensional. In figure 4 we investigate the orientations of these strings (excluding the smallest point-like structures from the analysis, including all structures in $E_{x}$ ). As expected, $E_{y}$ and $E_{z}$ are extended along the $x$-axis, $B_{y}$ and $B_{z}$ along the $t$-axis, with $B_{x}$ extended along both the $x$ and $t$ axes. 


\section{Conclusions}

We have suggested that, by introducing a carefully tuned field, $\theta$, it is possible to diagonalise the gauge links within a Wilson Loop without introducing additional path integrals or dynamical variables, giving a $U(1)^{N_{C}-1}$ Abelian theory (a CDG decomposition) which can be used to calculate the string tension. This theory can be studied numerically, and modelled theoretically. As expected, the coloured fields do not contribute to confinement. There may be certain topological singularities within $\theta$ which contribute to the string tension, giving characteristic structures appearing in the Abelian field strength tensor. We have confirmed numerically that the topological term accounts for all of the string tension, and that the structures within the field strength have the same dimensionality and directions as expected from the model.

\section{Acknowledgements}

Numerical calculations used servers at Seoul National University. Funding was provided by the BK21 program of the NRF, Republic of Korea. The research of W. Lee is supported by the Creative Research Initiatives Program (2012-0000241) of the NRF grant funded by the Korean government (MEST). W. Lee would like to acknowledge the support from KISTI supercomputing center through the strategic support program for the supercomputing application research [No. KSC-2011-G2-06]. YMC is supported in part by NRF grant (2012-002-134) funded by MEST.

\section{References}

[1] N. Cundy, W. Lee, J. Leem, and Y. Cho PoS LATTICE2012 (2012) 213, [arXiv: 1211.0664 ]; N. Cundy, Y. Cho, W. Lee, and J. Leem arXiv:1307. 3085.

[2] A. Kronfeld, M. Laursen, G. Schierholz, and U.-J. Wiese Phys. Lett. B 198 (1987), no. 4516 - 520; T. Suzuki and I. Yotsuyanagi Phys. Rev. D42 (1990) 4257-4260; P. de Forcrand and M. Pepe Nucl. Phys. B598 (2001) 557-577, [hep-lat/ 0008016 ]; J. D. Stack, S. D. Neiman, and R. J. Wensley Phys. Rev. D50 (1994) 3399-3405, [hep-lat/9404014]; H. Shiba and T. Suzuki Phys. Lett. B351 (1995) 519-527, [hep-lat/9408004]; J. Greensite Prog. Part. Nucl. Phys. 51 (2003) 1, [hep-lat/0301023].

[3] Y. M. Cho Phys. Rev. D 21 (1980) 1080; Y. M. Cho Phys. Rev. D 23 (1981) 2415; Y. Duan and M. Ge Sci. Sinica 11 (1979) 1072; L. Faddeev and A. Niemi Phys. Rev. Lett. 82 (1999) 1624, [hep-th/9807069]; S. Shabanov Phys. Lett. B 458 (1999) 322, [hep-th/ 9903223 ].

[4] K.-I. Kondo, T. Murakami, and T. Shinohara Prog.Theor.Phys. 115 (2006) 201-216, [hep-th/ 0504107$]$; K.-I. Kondo, A. Shibata, T. Shinohara, and S. Kato Phys. Rev. D83 (2011) 114016, [arXiv:1007.2696]; A. Shibata et al. POS LATTICE-2007 (2007) 331, [arXiv:0710.3221].

[5] K. G. Wilson in New Developments in Quantum Field Theory and Statistical Mechanics (M. Levy and P. Mitter, eds.), Plenum, New York, 1977.

[6] M. Lüscher and P. Weisz Commun Math Phys 97 (1985) 59; G. P. Lepage and P. B. Mackenzie Phys. Rev. D 48 (Sep, 1993) 2250-2264; J. Snippe Nucl. Phys B498 (1997) 347. 